\title{
QUEEN'S
UNIVERSITY
BELFAST
}

\section{Modifiable lifestyle factors associated with risk of sessile serrated polyps, conventional adenomas, and hyperplastic polyps}

Davenport, J. R., Su, T., Zhao, Z., Coleman, H. G., Smalley, W. E., Ness, R. M., Zheng, W., \& Shrubsole, M. J. (2016). Modifiable lifestyle factors associated with risk of sessile serrated polyps, conventional adenomas, and hyperplastic polyps. Gut. https://doi.org/10.1136/gutjnl-2016-312893

\section{Published in:}

Gut

\section{Document Version:}

Peer reviewed version

Queen's University Belfast - Research Portal:

Link to publication record in Queen's University Belfast Research Portal

\section{Publisher rights}

Copyright 2016 BMJ Publishing Group Limited.

This work is made available online in accordance with the publisher's policies. Please refer to any applicable terms of use of the publisher.

\section{General rights}

Copyright for the publications made accessible via the Queen's University Belfast Research Portal is retained by the author(s) and / or other copyright owners and it is a condition of accessing these publications that users recognise and abide by the legal requirements associated with these rights.

Take down policy

The Research Portal is Queen's institutional repository that provides access to Queen's research output. Every effort has been made to ensure that content in the Research Portal does not infringe any person's rights, or applicable UK laws. If you discover content in the Research Portal that you believe breaches copyright or violates any law, please contact openaccess@qub.ac.uk. 
Modifiable lifestyle factors associated with risk of sessile serrated polyps and conventional adenomas

James R. Davenport ${ }^{1,2}$, Timothy $\mathrm{Su}^{1}$, Zhiguo Zhao ${ }^{1,3,4}$, Helen G. Coleman ${ }^{5}$, Walter E. Smalley², Reid M. Ness ${ }^{2,6}$, Wei Zheng ${ }^{1,3,7}$, and Martha J. Shrubsole ${ }^{1,3,7^{*}}$

\section{Author Affiliations:}

${ }^{1}$ Department of Medicine, Division of Epidemiology, Vanderbilt University School of Medicine, Nashville, TN, USA.

2 Department of Medicine, Division of Gastroenterology, Hepatology, and Nutrition, Vanderbilt University School of Medicine, Nashville, TN, USA.

${ }^{3}$ Vanderbilt-Ingram Cancer Center, Vanderbilt University School of Medicine, Nashville, TN, USA.

${ }^{4}$ Department of Biostatistics, Vanderbilt University School of Medicine, Nashville, TN, USA. ${ }^{5}$ Cancer Epidemiology and Health Services Research Group, Centre for Public Health, Queen's University Belfast, Belfast, Northern Ireland.

${ }^{6}$ Gastroenterology Section, Department of Veterans Affairs, Tennessee Valley Healthcare System, Nashville, TN, USA.

${ }^{7}$ Geriatric Research, Education and Clinical Center (GRECC), Department of Veterans Affairs, Tennessee Valley Healthcare System, Nashville, TN, USA.

\section{*Corresponding Author and Guarantor:}

Martha J. Shrubsole, Division of Epidemiology, Department of Medicine, Vanderbilt University Medical Center, 2525 West End Avenue Room 837-A, Nashville, TN 37203, USA. Email: martha.shrubsole@vanderbilt.edu.

Word Count: 4053 


\section{ABSTRACT}

Objective: To identify modifiable factors associated with sessile serrated polyps (SSPs), and compare the association of these factors to conventional adenomas (ADs) and hyperplastic polyps (HPs).

Design: We utilized data from the Tennessee Colorectal Polyp Study, a colonoscopy-based case-control study. Included were $214 \underline{\text { SSP }}$ cases, $1779 \underline{\text { AD }}$ cases, 560 HP cases and 3851 polyp-free controls.

Results: Cigarette smoking was associated with increased risk for all polyps and was stronger for $\underline{\mathrm{SSP}} \mathrm{s}$ than for $\underline{\mathrm{ADs}}$ (OR 1.74. 95\% Cl: $1.16-2.62$, for current vs. never, $\mathrm{p}_{\text {trend }}=0.008$ ). Current regular use of nonsteroidal anti-inflammatories (NSAID) was associated with a $40 \%$ reduction in SSPs risk in comparison to never-users (OR $0.68,95 \% \mathrm{Cl} 0.48-0.96, \mathrm{p}_{\text {trend }}=0.03$ ), similar to the association with $\underline{\mathrm{AD}}$. Red meat intake was strongly associated with $\underline{\mathrm{SSP}} \mathrm{s}$ risk (OR $2.59,95 \% \mathrm{Cl}$ 1.41-4.74 for highest vs. lowest intake, $p_{\text {trend }}<0.001$ ) and the association with $\underline{\text { SPP }}$ was stronger than with $\underline{A D}$ ( $p_{\text {trend }}=0.003$ ). Obesity, folate intake, fiber intake, and fat intake were not associated with SSP risk after adjustment for other factors. Exercise, alcohol use, and calcium intake were not associated with risk for SSPs.

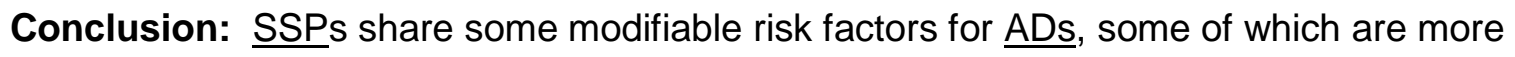
strongly associated with $\underline{\mathrm{SSP} s}$ than $\underline{\mathrm{ADs}}$. Thus, preventive efforts to reduce risk for $\underline{\mathrm{ADs}}$ may also be applicable to SSPs. Additionally, SSPs have some distinctive risk factors. Future studies should evaluate the preventive strategies for these factors. The findings from this study also contribute to an understanding of the etiology and biology of SSPs.

\section{SUMMARY}




\section{What is already known about this subject?}

- Most colorectal cancers are derived from two separate precursor pathways: a conventional adenoma-carcinoma pathway and a serrated pathway.

- Lifestyle factors such as cigarette smoking and increased red meat intake are known risk factors for conventional colorectal adenomas.

- Risk factors for sessile serrated polyps are less known, given their recent consensus regarding their defined pathology.

\section{What are the new findings?}

- Sessile serrated/polyps share some risk factors with conventional adenomas, and other risk factors are unique to sessile serrated polyps.

- Regular use of NSAIDs is associated with a reduction in risk of sessile serrated adenomas/polyps in addition to conventional adenomas.

- Red meat intake is strongly associated with increased risk of sessile serrated polyps in addition to conventional adenomas.

\section{How might it impact clinical practice in the foreseeable future?}

- Given that SSPs are difficult to detect and may accelerate to a dysplastic state quicker than conventional adenomas, primary prevention of sessile serrated adenomas/polyps through lifestyle modification may be an important strategy

- Preventive efforts to reduce risk factors in conventional adenomas may also be applicable to sessile serrated adenomas/polyps.

Keywords: Sessile serrated polyp, colorectal, adenoma, risk factors, etiology 


\section{INTRODUCTION}

Two distinct pathways to colorectal carcinogenesis have been identified. Well known is the conventional adenoma (AD)-carcinoma pathway, which involves the progression of nonadvanced tubular adenomas to larger or villous lesions with potential to develop into an invasive carcinoma [1]. In contrast, the more recently recognized serrated pathway is thought to originate from hyperplastic polyps (HPs), and transition to distinct traditional serrated adenomas or sessile serrated polyps (SSPs) prior to progression to dysplasia and carcinoma [2]. SSPS, although comprising only 4-9\% of all polyps discovered on endoscopy, may represent the origin for $20-35 \%$ of all CRCs, particularly those with microsatellite-instable (MSI-high) or CpG-island methylator phenotype (CIMP-high) features [3-5]. Unlike ADs, which are diffusely distributed,

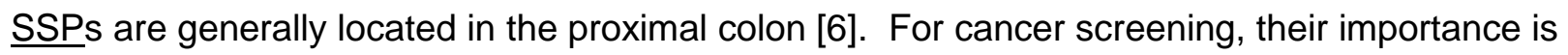
highlighted by new data, concluding that the decline of cancer incidence over 30 years has corresponded primarily to distal CRC lesions, while the comparative rate of decline of proximal $\mathrm{CRC}$ is 4-7 times less [7]. Furthermore, a sizeable proportion of interval CRCs, or cancers discovered between appropriate CRC screening intervals, are proximal and likely to have originated from SSPs which have either been missed, incompletely resected, or have rapidly progressed to a carcinogenic state[4,8,9].

Few studies have evaluated risk factors of SSPs due to challenges involved in their evolving histological definition and the relative rarity of these polyps. For multiple reasons, studies to date have often clustered HPs and SSPs into a collective 'serrated polyp' group, despite differences in malignant potential between the lesions. Similar to studies which evaluated risk for $\underline{A D s}$, a few studies found risk for serrated polyps was associated with cigarette smoking [1014], obesity [10-12], Type II diabetes mellitus [11], a family history of CRC [12], age [11,13], higher education [13], and nonsteroidal anti-inflammatory drug (NSAID) use [10,14]. Even fewer studies have evaluated the association of dietary factors with the risk of serrated polyps. 
These studies observed that red meat intake may be associated with increased risk in distal, but not proximal, serrated polyps [10]. However, most of these serrated polyp studies are limited by the sample size and/or the likely grouping of HPs and SSPs. Given the possibility that endoscopy may not reduce mortality of proximal CRCs and that SSPs may be the primary precursor lesion for these tumors, there is a compelling need to assess modifiable lifestyle factors which may be associated with $\underline{\text { SSPS }}$ and to compare the associations with risk for ADs and HPs.

We sought to conduct a comprehensive analysis of modifiable lifestyle risk factors which may affect SSP risk, and subsequently compare the associations between $\underline{\mathrm{ADS}}, \mathrm{HPS}$, and $\underline{\mathrm{SSP}}$. We utilized the Tennessee Colorectal Polyp Study (TCPS), a large case-control, colonoscopybased study, which has standardized assessment of SSPs, $\underline{\text { ADs }}$, and HPs. Our goal was to understand the etiology and develop a risk factor index to evaluate the joint contribution of risk factors to risk of SSPs and other polyps and to further compare risk factors between $\underline{\text { SPPs }}$ and $\underline{A D s}$ and HPs. This comparison may provide insight into the common and varied etiology of colorectal polyps.

\section{DESIGN}

\section{Study design and population}

TCPS is a colonoscopy-based case-control study conducted from February 2003 to October 2010 in Nashville, TN. Further details regarding the methods used are previously described [15]. For individuals 40-75 years of age, candidacy was discussed and consent obtained if the subject met eligibility standards. Ineligibility for the study was defined as any candidate having a history of inflammatory bowel disease (IBD) or if IBD was discovered on colonoscopy; any known family history of hereditary CRC syndromes; any history of cancer except for non- 
melanoma skin cancer; any previous colectomy; any diagnosis of adenomas on previous colonoscopy or surgical resection. Colonoscopies were conducted as part of routine care by trained gastroenterologists. Institutional approval for human subjects research was granted through the VUMC and VA Institutional Review Boards and the VA Research and Development Committee.

There were 12,585 candidates initially identified for participation in TCPS, with 7,621 participants (60.6\%) providing an informed written consent and participating in at least one component of the study. A majority of participants (90.5\%) were recruited prior to colonoscopy, and the remaining were recruited post-colonoscopy. Among the participants, 7,396 were diagnosed with $\underline{\mathrm{ADS}}, \mathrm{HPS}, \underline{\mathrm{SSP}} \mathrm{s}$ or no polyps, and were thus eligible for this analysis. The current analysis is based on a total of 6,404 eligible participants (86.6\%) who completed a telephone interview (median time to interview was 13 days). For dietary analyses, analyses were further limited to participants who also completed a 108-item food frequency questionnaire (FFQ; median time to FFQ return was 23 days) and reported daily consumption of at least 600 kcal/day (5,398 individuals; 84.3\%) [16].

\section{Data collection}

Following the colonoscopy, interviewers used a standard telephone interview to obtain information relating to the participant's demographics, medication use, family history, and other lifestyle factors. Detailed questions regarding status, intensity, duration, age of cessation, and age of initiation of tobacco use were asked, with current smokers defined as one cigarette consumed daily for each of the past six months and over 100 cigarettes within their lifetime. Former smokers must have quit more than one year prior to their procedure. Any reported smoking in the last 12 months placed them in the current smoking group. Current alcohol use was defined as five or more alcoholic beverages per week over the past year. Former users did 
not meet this criteria for 12 months or greater prior to their procedure. Body Mass Index (BMI) was calculated from self-reported height and weight. Regular exercise was defined as nonoccupational exercise for at least two hours per week over a six month period within the past decade, with further breakdown using metabolic equivalent of task (MET) hours per week. For defining current or former NSAID use, current users took NSAIDs at least three times weekly for the past 12 months, while former users took NSAIDs three times weekly for 12 months over the past 15 years, but without use within the last 12 months. Dietary information was selfadministered using the FFQ, except in the case of red meat intake, which was obtained during the telephone interview in methods previously described [15]. Dietary components in the FFQ which were examined include daily intake of total energy (kilocalories), fiber (g/day), dietary folate equivalents (DFE, $\mu \mathrm{g} /$ day), calcium (mg/day), and fat (g/day) as previously described [16].

\section{Classification of case groups}

All participants were recruited between 2002 and 2010, during which SSPs were not uniformly recognized in clinical practice, nor was a standard pathology definition developed. As a result, the potential for misclassification of SSPs as another type of polyp (e.g. hyperplastic) was substantial. To overcome this limitation of the original clinical diagnosis, we newly reviewed all polyps from all study participants, regardless of the initial clinical diagnosis to standardize all diagnoses. The study pathologist and a senior GI clinical and research pathologist established a consensus on application of the diagnostic criteria from expert panel standards (at least one distorted, dilated, or horizontally branched crypt within the polyp) by joint review of cases [3]. In addition, the study pathologist identified about $10 \%$ of cases in which there was a potential for disagreement and both pathologists reviewed those cases to reach consensus. Based on the pathology diagnosis, we excluded individuals who were found to have evidence of $C R C(n=26)$ or traditional serrated adenomas $(n=12)$, due to limited statistical power. Control participants underwent a full colonoscopy, with evidence of reaching the cecum and complete colon 
visualization without a notation of polyps. Visualization of the ileocecal valve and/or appendiceal orifice was achieved for $98.8 \%$ of polyp cases. The HP cases had one or more HPs without any synchronous $\underline{A D}$ or SSP. The $\underline{A D}$ cases had one or more tubular, tubulovillous, or villous $\underline{A D}$ with or without dysplasia and with or without synchronous HPs. The $\underline{\text { SSP }}$ cases had one or more SSPs, with or without synchronous HPs and ADs.

\section{Statistical analysis}

Descriptive comparisons between case and control groups were calculated using general linear models (for continuous variables) or Mantel-Haenszel $x^{2}$ testing (for categorical variables), with adjustments in most comparisons for age (based on categories grouping individuals into 5-year age categories from 40-75), and sex. Dietary intake quartiles were derived from intake levels among controls. Initial assessment of risk for case-control and case-case comparisons was completed using multinomial logistic regression modeling which included each case and control group in each model to allow direct comparison of each case group. Models were adjusted for sex, age of the participant (based on the categories listed above), year of the colonoscopy, educational attainment, study site, cigarette smoking, and NSAID use. Additional models, which included dietary factors, were adjusted for total energy intake. In order to test for trends, we treated categorical variables as continuous factors in the model. To assess whether factors had an independent association with polyp risk, we conducted further analysis in which factors which were statistically significantly associated in initial models were included in a subsequent multinomial logistic regression model in which they were mutually adjusted for each other. All statistical analyses were completed using $R$ Version 3. P values of $\leq 0.05$ (2-sided probability) were considered statistically significant in all analyses.

\section{RESULTS}


Demographic characteristics of each of the four groups examined (no-polyp controls, $\underline{A D s}$, HPs, and SSAs) are shown in Table 1. No significant differences were found between controls and case groups in comparing the procedure site, race, indication for the colonoscopy, or family history of CRC. Age ( $\left.p_{\text {heterogeneity }}<0.001\right)$, sex $\left(p_{\text {heterogeneity }}<0.001\right)$, educational attainment ( $\left.p_{\text {heterogeneity }}<0.001\right)$, household income $\left(p_{\text {heterogeneity }}=0.002\right)$ and energy intake $\left(p_{\text {heterogeneity }}=0.009\right)$ were significantly different between groups. 
Table 1: Characteristics of the Study Participants, the Tennessee Colorectal Polyp Study.

\begin{tabular}{|c|c|c|c|c|c|}
\hline Characteristic & $\begin{array}{c}\text { No polyp } \\
\text { Controls } \\
n=3851\end{array}$ & $\begin{array}{c}\text { Hyperplastic } \\
\text { Polyps } \\
n=560\end{array}$ & $\begin{array}{c}\text { Conventional } \\
\text { Adenomas } \\
n=1779\end{array}$ & $\begin{array}{c}\text { Sessile Serrated } \\
\text { Polyps } \\
n=214\end{array}$ & Pheterogeneity \\
\hline \multicolumn{6}{|l|}{ Study Site of Procedure ${ }^{a}$} \\
\hline Vanderbilt University MC & 74.8 & 70 & 74.5 & 74.6 & 0.07 \\
\hline VA-Nashville Campus & 25.2 & 30 & 25.6 & 25.4 & \\
\hline Age (years) & $57.2 \pm 7.7$ & $56.8 \pm 7.1$ & $59.0 \pm 7.4$ & $57.8 \pm 7.7$ & $<0.001$ \\
\hline Sex (\% Female) & 44.8 & 36.4 & 27.5 & 36 & $<0.001$ \\
\hline Race (\% Caucasian) & 87 & 89.3 & 87 & 89.7 & 0.34 \\
\hline Family History of Colorectal Cancer (\%) ${ }^{\mathrm{a}}$ & 9 & 8.6 & 9.4 & 11.9 & 0.44 \\
\hline \multicolumn{6}{|l|}{ Indication for Colonoscopy (\%) ${ }^{\mathrm{a}}$} \\
\hline Average Risk Screening & 56.9 & 54.3 & 55.7 & 57.9 & 0.2 \\
\hline Family History of Colorectal Cancer & 12.6 & 13.7 & 12.5 & 16.2 & \\
\hline Diagnostic/Follow Up & 22.8 & 21.6 & 23.6 & 15.4 & \\
\hline Other & 7.8 & 10.4 & 8.2 & 10.5 & \\
\hline \multicolumn{6}{|l|}{ Educational Attainment (\%)a } \\
\hline High School or Less & 23.4 & 29.5 & 28.3 & 25 & $<0.001$ \\
\hline Some College & 28.4 & 28.3 & 28.5 & 27.4 & \\
\hline College Graduate & 21.4 & 21.9 & 22.1 & 27.1 & \\
\hline Graduate/Professional School & 26.8 & 20.3 & 21.1 & 20.5 & \\
\hline
\end{tabular}




\begin{tabular}{|c|c|c|c|c|c|}
\hline Characteristic & $\begin{array}{l}\text { No polyp } \\
\text { Controls } \\
n=3851\end{array}$ & $\begin{array}{c}\text { Hyperplastic } \\
\text { Polyps } \\
n=560\end{array}$ & $\begin{array}{c}\text { Conventional } \\
\text { Adenomas } \\
n=1779\end{array}$ & $\begin{array}{c}\text { Sessile Serrated } \\
\frac{\text { Polyps }}{n=214}\end{array}$ & Pheterogeneity \\
\hline \multicolumn{6}{|l|}{ Household Income (\%) } \\
\hline Under $\$ 15,000$ & 7.8 & 9.6 & 10.6 & 6.7 & 0.002 \\
\hline$\$ 15,001-\$ 30,000$ & 14.1 & 17.9 & 16.2 & 15.6 & \\
\hline$\$ 30,001-\$ 50,000$ & 20.4 & 17.2 & 19.8 & 23.3 & \\
\hline Over $\$ 50,000$ & 57.7 & 55.3 & 53.5 & 54.4 & \\
\hline Daily Energy Intake (kcal) & 1845 & 1938 & 1912 & 1820 & 0.009 \\
\hline
\end{tabular}

${ }^{s}$ Standardized by age (using ages grouped into 5 year categories starting at age 40) and sex. 


\section{Evaluation of modifiable non-dietary factors and polyp risk}

Cigarette smoking status, duration, and intensity were associated with increased polyp risk for all case types (Table 2). In case-case comparisons, smoking was more strongly associated with SSPs than $\underline{A D s}$ for all measures of smoking (e.g. OR 1.74, 95\% Cl 1.16-2.62 for current vs. never smokers, $\left.p_{\text {trend }}=0.008\right)$. Obesity $\left(B M I \geq 30 \mathrm{~kg} / \mathrm{m}^{2}\right)$ was associated with a $30 \%-50 \%$ increased risk of polyps and risk did not significantly differ between polyp types. In comparison to those who never regularly used NSAIDs, current regular use of NSAIDs was associated with a decreased risk of $\underline{\text { SSPS }}\left(\mathrm{OR} 0.62,95 \% \mathrm{Cl} 0.62-0.85\right.$ for $\underline{\mathrm{SSP}}$ cases vs. controls, $\mathrm{p}_{\text {trend }}=0.003$ ) and $\underline{A D s}\left(p_{\text {trend }}<0.001\right)$ and but not HPs, and risk reduction was dose-dependent for years of use for both $\underline{A D s}\left(p_{\text {trend }}=0.02\right)$ and SSPs $\left(p_{\text {trend }}<0.001\right)$. In addition, NSAID use of more than 10 years was more strongly associated with reduced risk of SSPs than $\underline{\text { ADs }}$ (OR 0.53, 95\% $0.31-0.92$ for $>10$ years vs never regular use). Use of NSAIDs more than 7 times a week were also associated with reduced risks of SSP and AD. Alcohol use and exercise were not associated with risk of any polyp type. 
Table 2: Associations between Modifiable Non-Dietary Factors and Polyp Risk; the Tennessee Colorectal Polyp Study.

\begin{tabular}{|c|c|c|c|c|c|c|c|c|c|c|}
\hline \multirow{3}{*}{$\begin{array}{l}\text { Risk } \\
\text { Factor }\end{array}$} & \multicolumn{7}{|c|}{ Case-Control Comparisons } & \multicolumn{3}{|c|}{ Case-Case Comparisons } \\
\hline & \multirow{2}{*}{$\begin{array}{c}\begin{array}{c}\text { No } \\
\text { polyp } \\
\text { Controls }\end{array} \\
\mathrm{n}\end{array}$} & \multicolumn{2}{|r|}{$\begin{array}{l}\text { Hyperplastic } \\
\text { Polyps } \\
\text { (HP) }\end{array}$} & \multicolumn{2}{|c|}{$\begin{array}{l}\text { Conventional } \\
\text { Adenomas } \\
\text { (AD) }\end{array}$} & \multicolumn{2}{|c|}{$\begin{array}{l}\text { Sessile Serrated } \\
\text { Polyps } \\
\text { (SSP) }\end{array}$} & \multirow{2}{*}{$\begin{array}{c}\underline{\text { AD }} \text { vs. HP } \\
\text { OR }(95 \% \mathrm{Cl})^{\mathrm{a}}\end{array}$} & \multirow{2}{*}{$\begin{array}{l}\text { SSP vs. HP } \\
\text { OR }(95 \% \mathrm{Cl})^{\mathrm{a}}\end{array}$} & \multirow{2}{*}{$\begin{array}{l}\underline{\text { SSP }} \text { vs. } \underline{\mathrm{AD}} \\
\text { OR }(95 \% \mathrm{Cl})^{\mathrm{a}}\end{array}$} \\
\hline & & $\mathrm{n}$ & OR $(95 \% \mathrm{Cl})^{\mathrm{a}}$ & $\mathrm{n}$ & OR $(95 \% \mathrm{Cl})^{a}$ & $\mathrm{n}$ & OR $(95 \% \mathrm{Cl})^{a}$ & & & \\
\hline \multicolumn{11}{|c|}{ Cigarette Smoking Status $^{b}$} \\
\hline Never & 2042 & 170 & 1.00 (ref) & 685 & 1.00 (ref) & 76 & 1.00 (ref) & 1.00 (ref) & 1.00 (ref) & 1.00 (ref) \\
\hline Former & 1313 & 215 & $2.10(1.67,2.64)$ & 655 & $1.29(1.12,1.49)$ & 71 & $1.46(1.01,2.10)$ & $0.61(0.48,0.79)$ & $0.69(0.46,1.05)$ & $1.13(0.77,1.65)$ \\
\hline Current & 490 & 175 & $4.60(3.54,5.98)$ & 437 & $2.46(2.06,2.94)$ & 66 & $4.29(2.87,6.40)$ & $0.53(0.41,0.71)$ & $0.93(0.59,1.46)$ & $1.74(1.16,2.62)$ \\
\hline $\mathbf{P}_{\text {trend }}$ & & & $<0.001$ & & $<0.001$ & & $<0.001$ & $<0.001$ & 0.73 & 0.008 \\
\hline \multicolumn{11}{|c|}{ Cigarette Smoking Duration (years) } \\
\hline Never & 2042 & 170 & 1.00 (ref) & 685 & 1.00 (ref) & 76 & 1.00 (ref) & 1.00 (ref) & 1.00 (ref) & 1.00 (ref) \\
\hline $1-15$ & 559 & 61 & $1.45(1.06,1.99)$ & 207 & $1.08(0.89,1.32)$ & 25 & $1.21(0.73,2.01)$ & $0.75(0.53,1.05)$ & $0.84(0.47,1.49)$ & $1.12(0.67,1.89)$ \\
\hline $15-25$ & 353 & 71 & $2.61(1.91,3.58)$ & 166 & $1.26(1.01,1.57)$ & 19 & $1.29(0.72,2.32)$ & $0.48(0.34,0.68)$ & $0.49(0.26,0.94)$ & $1.02(0.56,1.86)$ \\
\hline $25-35$ & 392 & 92 & $3.09(2.31,4.15)$ & 252 & $1.85(1.51,2.25)$ & 40 & $3.18(2.06,4.91)$ & $0.60(0.44,0.82)$ & $1.03(0.63,1.69)$ & $1.72(1.11,2.69)$ \\
\hline$>35$ & 498 & 166 & $4.73(3.59,6.22)$ & 462 & $2.28(1.90,2.73)$ & 52 & $3.30(2.87,5.08)$ & $0.53(0.41,0.71)$ & $0.70(0.43,1.13)$ & $1.45(0.94,2.24)$ \\
\hline $\mathbf{P}_{\text {trend }}$ & & & $<0.001$ & & $<0.001$ & & $<0.001$ & $<0.001$ & 0.28 & 0.03 \\
\hline \multicolumn{11}{|c|}{ Cigarette Smoking Intensity (pack-years) ${ }^{b}$} \\
\hline Never & 2042 & 170 & 1.00 (ref) & 685 & 1.00 (ref) & 76 & 1.00 (ref) & 1.00 (ref) & 1.00 (ref) & 1.00 (ref) \\
\hline $1-9$ & 588 & 83 & $1.94(1.46,2.59)$ & 239 & $1.32(1.09,1.59)$ & 31 & $1.55(0.97,2.46)$ & $0.68(0.50,0.93)$ & $0.80(0.47,1.34)$ & $1.17(0.73,1.89)$ \\
\hline $10-29$ & 588 & 142 & $3.12(2.41,4.04)$ & 297 & $1.32(1.10,1.58)$ & 36 & $1.76(1.13,2.75)$ & $0.42(0.32,0.56)$ & $0.56(0.34,0.92)$ & $1.34(0.85,2.11)$ \\
\hline
\end{tabular}




\begin{tabular}{|c|c|c|c|c|c|c|c|c|c|c|}
\hline \multirow{3}{*}{$\begin{array}{l}\text { Risk } \\
\text { Factor }\end{array}$} & \multicolumn{7}{|c|}{ Case-Control Comparisons } & \multicolumn{3}{|c|}{ Case-Case Comparisons } \\
\hline & \multirow{2}{*}{$\begin{array}{c}\begin{array}{c}\text { No } \\
\text { polyp } \\
\text { Controls }\end{array} \\
\mathrm{n}\end{array}$} & \multicolumn{2}{|r|}{$\begin{array}{l}\text { Hyperplastic } \\
\text { Polyps } \\
\text { (HP) }\end{array}$} & \multicolumn{2}{|c|}{$\begin{array}{l}\frac{\text { Conventional }}{\text { Adenomas }} \\
\text { (AD) }\end{array}$} & \multicolumn{2}{|c|}{$\begin{array}{l}\text { Sessile Serrated } \\
\text { Polyps } \\
\text { (SSP) }\end{array}$} & \multirow{2}{*}{$\begin{array}{c}\underline{\text { AD }} \text { vs. HP } \\
\text { OR }(95 \% \mathrm{Cl})^{\mathrm{a}}\end{array}$} & \multirow{2}{*}{$\begin{array}{l}\text { SSP vs. HP } \\
\text { OR }(95 \% \mathrm{Cl})^{\mathrm{a}}\end{array}$} & \multirow{2}{*}{$\begin{array}{l}\underline{\text { SSP }} \text { vs. } \underline{\text { AD }} \\
\text { OR }(95 \% \mathrm{Cl})^{\mathrm{a}}\end{array}$} \\
\hline & & $\mathrm{n}$ & OR $(95 \% \mathrm{Cl})^{\mathrm{a}}$ & $\mathrm{n}$ & OR $(95 \% \mathrm{Cl})^{\mathrm{a}}$ & $\mathrm{n}$ & OR $(95 \% \mathrm{Cl})^{a}$ & & & \\
\hline$\geq 30$ & 651 & 164 & $3.18(2.43,4.16)$ & 549 & $2.10(1.77,2.48)$ & 69 & $3.21(2.14,4.81)$ & $0.66(0.50,0.87)$ & $1.01(0.63,1.60)$ & $1.53(1.01,2.31)$ \\
\hline $\mathbf{P}_{\text {trend }}$ & & & $<0.001$ & & $<0.001$ & & $<0.001$ & $<0.001$ & 0.70 & 0.04 \\
\hline
\end{tabular}

\section{Recency of Cigarette Smoking (years) ${ }^{b}$}

\begin{tabular}{|c|c|c|c|c|c|c|c|c|c|c|}
\hline Current & 490 & 175 & 1.00 (ref) & 437 & 1.00 (ref) & 66 & 1.00 (ref) & 1.00 (ref) & 1.00 (ref) & 1.00 (ref) \\
\hline Quit <10 & 245 & 63 & $0.67(0.48,0.94)$ & 158 & $0.71(0.55,0.91)$ & 13 & $0.38(0.20,0.71)$ & $1.06(0.75,1.52)$ & $0.57(0.29,1.12)$ & $0.54(0.28,1.01)$ \\
\hline Quit 10-20 & 307 & 69 & $0.64(0.46,0.88)$ & 174 & $0.60(0.47,0.76)$ & 24 & $0.55(0.33,0.91)$ & $0.94(0.67,1.33)$ & $0.86(0.49,1.51)$ & $0.91(0.54,1.53)$ \\
\hline Quit >20 & 761 & 83 & $0.29(0.22,0.40)$ & 323 & $0.42(0.35,0.52)$ & 34 & $0.24(0.15,0.39)$ & $1.44(1.04,1.99)$ & $0.81(0.47,1.40)$ & $0.56(0.34,0.92)$ \\
\hline Never & 2042 & 170 & $0.21(0.16,0.27)$ & 685 & $0.40(0.33,0.48)$ & 76 & $0.23(0.15,0.34)$ & $1.90(1.44,2.50)$ & $1.08(0.69,1.70)$ & $0.57(0.38,0.86)$ \\
\hline $\mathbf{P}_{\text {trend }}$ & & & $<0.001$ & & $<0.001$ & & $<0.001$ & $<0.001$ & 0.63 & 0.009 \\
\hline
\end{tabular}

Regular Alcohol Use $e^{\mathrm{b}, \mathrm{c}}$

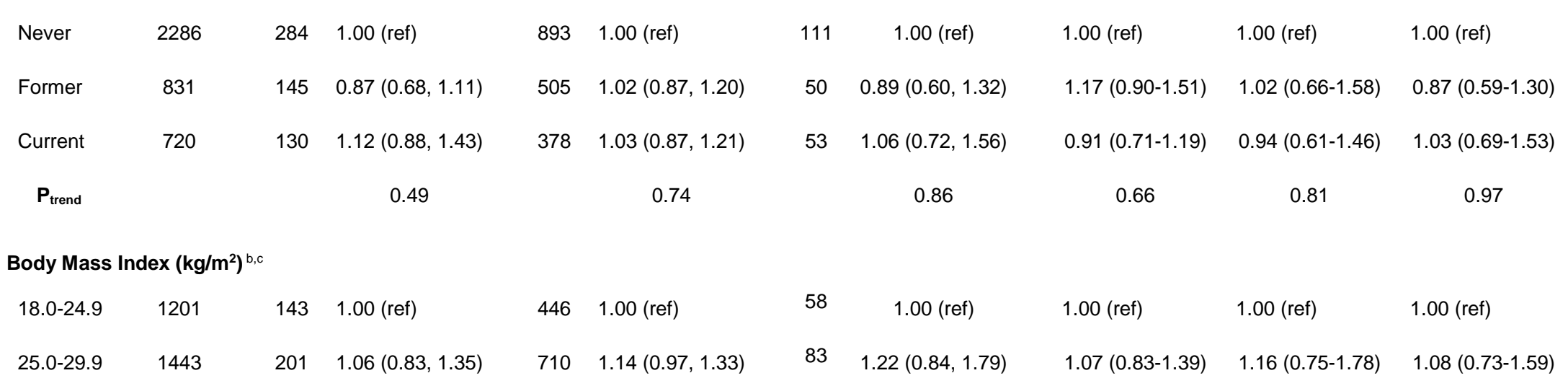




\begin{tabular}{|c|c|c|c|c|c|c|c|c|c|c|}
\hline \multirow{3}{*}{$\begin{array}{l}\text { Risk } \\
\text { Factor }\end{array}$} & \multicolumn{7}{|c|}{ Case-Control Comparisons } & \multicolumn{3}{|c|}{ Case-Case Comparisons } \\
\hline & \multirow{2}{*}{$\begin{array}{c}\begin{array}{c}\text { No } \\
\text { polyp } \\
\text { Controls }\end{array} \\
n\end{array}$} & \multicolumn{2}{|r|}{$\begin{array}{l}\text { Hyperplastic } \\
\text { Polyps } \\
\text { (HP) }\end{array}$} & \multicolumn{2}{|c|}{$\begin{array}{l}\frac{\text { Conventional }}{\text { Adenomas }} \\
\text { (AD) }\end{array}$} & \multicolumn{2}{|c|}{$\begin{array}{c}\text { Sessile Serrated } \\
\text { Polyps } \\
\text { (SSP) }\end{array}$} & \multirow{2}{*}{$\begin{array}{c}\underline{\text { AD }} \text { vs. HP } \\
\text { OR }(95 \% \mathrm{Cl})^{\mathrm{a}}\end{array}$} & \multirow{2}{*}{$\begin{array}{l}\text { SSP vs. HP } \\
\text { OR }(95 \% \mathrm{Cl})^{\mathrm{a}}\end{array}$} & \multirow{2}{*}{$\begin{array}{l}\underline{\text { SSP vs. } \underline{\text { AD }}} \\
\text { OR }(95 \% \mathrm{Cl})^{\mathrm{a}}\end{array}$} \\
\hline & & $\mathrm{n}$ & OR $(95 \% \mathrm{Cl})^{\mathrm{a}}$ & $\mathrm{n}$ & OR $(95 \% \mathrm{Cl})^{a}$ & $\mathrm{n}$ & OR $(95 \% \mathrm{Cl})^{a}$ & & & \\
\hline$>30.0$ & 1189 & 215 & $1.49(1.17,1.90)$ & 611 & $1.34(1.13,1.57)$ & 72 & $1.51(1.02,2.24)$ & $0.90(0.69-1.16)$ & $1.02(0.66-1.58)$ & $1.13(0.76-1.69)$ \\
\hline$P_{\text {trend }}$ & & & $<0.001$ & & $<0.001$ & & 0.04 & 0.34 & 0.98 & 0.55 \\
\hline \multicolumn{11}{|c|}{ Regular Exercise $\mathrm{e}^{\mathrm{b}, \mathrm{c}}$} \\
\hline No & 1606 & 266 & 1.00 (ref) & 863 & 1.00 (ref) & 58 & 1.00 (ref) & 1.00 (ref) & 1.00 (ref) & 1.00 (ref) \\
\hline Yes & 2245 & 294 & $0.96(0.79,1.16)$ & 916 & $0.90(0.79,1.02)$ & 83 & $0.91(0.67,1.24)$ & $0.94(0.76-1.16)$ & $0.95(0.67-1.34)$ & $1.01(0.74-1.39)$ \\
\hline $\mathbf{P}_{\text {trend }}$ & & & 0.67 & & 0.11 & & 0.55 & 0.34 & 0.77 & 0.94 \\
\hline \multicolumn{11}{|c|}{ Regular Exercise Intensity (MET hours per week) } \\
\hline $0.1-10.5$ & 558 & 80 & 1.00 (ref) & 235 & 1.00 (ref) & 26 & 1.00 (ref) & 1.00 (ref) & 1.00 (ref) & 1.00 (ref) \\
\hline $10.5-21.0$ & 565 & 73 & $0.98(0.69,1.40)$ & 248 & $1.05(0.83,1.33)$ & 34 & $1.11(0.62,2.00)$ & $1.07(0.73,1.56)$ & $1.13(0.59,2.17)$ & $1.06(0.58,1.92)$ \\
\hline $21.0-36.2$ & 558 & 71 & $0.90(0.62,1.28)$ & 208 & $0.92(0.72,1.17)$ & 31 & $1.29(0.73,2.27)$ & $1.03(0.69,1.51)$ & $1.44(0.76,2.72)$ & $1.40(0.78,2.51)$ \\
\hline$>36.2$ & 563 & 68 & $0.83(0.58,1.19)$ & 222 & $0.91(0.72,1.16)$ & 23 & $0.82(0.44,1.53)$ & $1.10(0.74,1.63)$ & $0.99(0.50,1.98)$ & $0.90(0.48,1.70)$ \\
\hline $\mathbf{P}_{\text {trend }}$ & & & 0.27 & & 0.30 & & 0.71 & 0.69 & 0.78 & 0.96 \\
\hline \multicolumn{11}{|c|}{ Regular NSAID Use $^{c}$} \\
\hline Never & 1698 & 241 & 1.00 (ref) & 744 & 1.00 (ref) & 103 & 1.00 (ref) & 1.00 (ref) & 1.00 (ref) & 1.00 (ref) \\
\hline Former & 245 & 33 & $0.89(0.60,1.33)$ & 108 & $0.97(0.75,1.25)$ & 11 & $0.72(0.38,1.37)$ & $1.09(0.71-1.66)$ & $0.81(0.39-1.67)$ & $0.74(0.38-1.44)$ \\
\hline Current & 1881 & 267 & $0.92(0.76,1.12)$ & 784 & $0.79(0.69,0.90)$ & 82 & $0.62(0.46,0.85)$ & $0.86(0.69-1.05)$ & $0.68(0.48-0.96)$ & $0.79(0.57-1.09)$ \\
\hline $\mathbf{P}_{\text {trend }}$ & & & 0.41 & & $<0.001$ & & 0.003 & 0.14 & 0.03 & 0.15 \\
\hline
\end{tabular}




\begin{tabular}{|c|c|c|c|c|c|c|c|c|c|c|}
\hline \multirow{3}{*}{$\begin{array}{l}\text { Risk } \\
\text { Factor }\end{array}$} & \multicolumn{7}{|c|}{ Case-Control Comparisons } & \multicolumn{3}{|c|}{ Case-Case Comparisons } \\
\hline & \multirow{2}{*}{$\begin{array}{c}\begin{array}{c}\text { No } \\
\text { polyp } \\
\text { Controls }\end{array} \\
\mathrm{n}\end{array}$} & \multicolumn{2}{|r|}{$\begin{array}{l}\text { Hyperplastic } \\
\text { Polyps } \\
\text { (HP) }\end{array}$} & \multicolumn{2}{|c|}{$\begin{array}{l}\frac{\text { Conventional }}{\text { Adenomas }} \\
(\underline{A D})\end{array}$} & \multicolumn{2}{|c|}{$\begin{array}{l}\text { Sessile Serrated } \\
\text { Polyps } \\
\text { ( } \underline{\text { SSP) }}\end{array}$} & \multirow{2}{*}{$\begin{array}{c}\underline{\text { AD }} \text { vs. HP } \\
\text { OR }(95 \% \mathrm{Cl})^{\mathrm{a}}\end{array}$} & \multirow{2}{*}{$\begin{array}{l}\text { SSP vs. HP } \\
\text { OR }(95 \% \mathrm{Cl})^{\mathrm{a}}\end{array}$} & \multirow{2}{*}{$\begin{array}{l}\underline{\text { SSP }} \text { vs. } \underline{\text { AD }} \\
\text { OR }(95 \% \mathrm{Cl})^{\mathrm{a}}\end{array}$} \\
\hline & & $\mathrm{n}$ & OR $(95 \% \mathrm{Cl})^{\mathrm{a}}$ & $\mathrm{n}$ & OR $(95 \% \mathrm{Cl})^{\mathrm{a}}$ & $\mathrm{n}$ & OR $(95 \% \mathrm{Cl})^{\mathrm{a}}$ & & & \\
\hline \multicolumn{11}{|c|}{ Duration of Regular NSAID Use (years) ${ }^{c}$} \\
\hline $0-<1$ & 1698 & 241 & 1.00 (ref) & 744 & 1.00 (ref) & 103 & 1.00 (ref) & 1.00 (ref) & 1.00 (ref) & 1.00 (ref) \\
\hline $1-5$ & 979 & 122 & $0.83(0.65,1.05)$ & 374 & $0.78(0.66,0.91)$ & 48 & $0.76(0.53,1.09)$ & $0.94(0.73,1.21)$ & $0.92(0.61,1.38)$ & $0.98(0.67,1.41)$ \\
\hline $6-10$ & 618 & 106 & $1.11(0.86,1.44)$ & 272 & $0.85(0.71,1.01)$ & 27 & $0.62(0.39,0.97)$ & $0.76(0.58,1.00)$ & $0.56(0.34,0.91)$ & $0.73(0.46,1.16)$ \\
\hline$>10$ & 529 & 73 & $0.86(0.64,1.15)$ & 246 & $0.81(0.67,0.98)$ & 18 & $0.43(0.25,0.74)$ & $0.95(0.69,1.29)$ & $0.50(0.28,0.91)$ & $0.53(0.31,0.92)$ \\
\hline $\mathbf{P}_{\text {trend }}$ & & & 0.70 & & 0.02 & & $<0.001$ & 0.26 & 0.004 & 0.01 \\
\hline
\end{tabular}

Dose of Regular NSAID Use (times per week) ${ }^{c}$

\begin{tabular}{|c|c|c|c|c|c|c|c|c|c|c|}
\hline$\underline{0}$ & $\underline{1588}$ & $\underline{242}$ & 1.00 (ref) & $\underline{832}$ & 1.00 (ref) & $\underline{115}$ & 1.00 (ref) & 1.00 (ref) & 1.00 (ref) & 1.00 (ref) \\
\hline$\leq 7$ & $\underline{304}$ & $\underline{45}$ & $1.01(0.71-1.43)$ & $\underline{106}$ & $\underline{0.79(0.62-1.02)}$ & $\underline{11}$ & $\underline{0.56(0.29-1.07)}$ & $\underline{0.79(0.54-1.16)}$ & $\underline{0.56(0.28-1.13)}$ & $\underline{0.71(0.37-1.37)}$ \\
\hline$\underline{7}$ & $\underline{965}$ & $\underline{137}$ & $\underline{0.92(0.72-1.17)}$ & $\underline{453}$ & $\underline{0.83(0.71-0.97)}$ & $\underline{55}$ & $\underline{0.77(0.54-1.10)}$ & $\underline{0.90(0.70-1.16)}$ & $\underline{0.84(0.56-1.26)}$ & $\underline{0.93(0.65-1.34)}$ \\
\hline$\geq 7$ & $\underline{994}$ & $\underline{136}$ & $\underline{0.86(0.67-1.09)}$ & $\underline{388}$ & $\underline{0.75(0.64-0.88)}$ & $\underline{33}$ & $\underline{0.48(0.32-0.73)}$ & $\underline{0.88(0.68-1.13)}$ & $\underline{0.57(0.36-0.90)}$ & $\underline{0.64(0.42-0.98)}$ \\
\hline trend & & & $\underline{0.19}$ & & $\leq 0.001$ & & $\underline{0.001}$ & $\underline{0.30}$ & $\underline{0.03}$ & $\underline{0.08}$ \\
\hline
\end{tabular}

${ }^{a}$ Derived from multinomial logistic regression models which included all case and controls groups and adjusted for age (40-49, 50-59, 60-64, and 65+ years of age), sex, educational attainment, year of colonoscopy, and study site

${ }^{b}$ Additionally adjusted for NSAID use

${ }^{c}$ Additionally adjusted for cigarette smoking status 


\section{Evaluation of modifiable dietary factors and polyp risk}

Higher daily dietary intake of fiber was associated with a reduced risk of SSPs (OR 0.36, 95\% Cl 0.19-0.68 for highest vs. lowest intake quartile, $\left.p_{\text {trend }}=0.006\right) \underline{\text { but was not statistically }}$

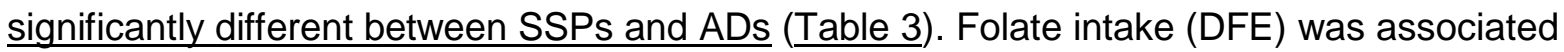
with an approximate $50 \%$ reduction in risk for all polyp types. Calcium intake was only associated with statistically significantly reduced risks of $\underline{A D s}$ and HPs, and was not associated with a statistically significantly reduced risk of SSPs although the associations were in the same direction and of similar magnitude. However, risk was not statistically significantly different between any of the case groups. Fat intake was associated with a strong dose-dependent three-fold increased risk of SSPs in comparison to controls (OR 3.09, 95\% Cl 1.24-7.72 for highest vs. lowest intake quartile, $\left.p_{\text {trend }}=0.01\right)$ and $\underline{A D}$ cases (OR 3.20, 95\% Cl 1.26-8.12 for highest vs. lowest intake quartile, $\mathrm{p}_{\text {trend }}=0.02$ ). Higher red meat intake was associated with all types of polyp risk, but displayed a particularly strong association with $\underline{\text { SP }}$ risk (OR 3.38, 95\% $\mathrm{Cl}$ 1.90-6.00 for highest vs. lowest intake quartile, $\left.\mathrm{p}_{\text {trend }}<0.001\right)$. In case-case comparisons, SSP risk was approximately two-fold greater than risks of either $\underline{\text { ADs }}$ or HPs for individuals consuming higher red meat intakes. 
Table 3: Associations between Modifiable Dietary Factors and Polyp Risk; the Tennessee Colorectal Polyp Study.

\begin{tabular}{|c|c|c|c|c|c|c|c|c|c|c|}
\hline \multirow{3}{*}{$\begin{array}{l}\text { Dietary } \\
\text { Intake } \\
\text { (per day) }\end{array}$} & \multicolumn{7}{|c|}{ Case-Control Comparisons } & \multicolumn{3}{|c|}{ Case-Case Comparisons } \\
\hline & \multirow{2}{*}{$\begin{array}{c}\begin{array}{c}\text { No } \\
\text { polyp } \\
\text { Controls }\end{array} \\
\mathrm{n}\end{array}$} & \multicolumn{2}{|r|}{$\begin{array}{l}\text { Hyperplastic } \\
\text { Polyps } \\
\text { (HP) }\end{array}$} & \multicolumn{2}{|c|}{$\begin{array}{l}\text { Conventional } \\
\text { Adenomas } \\
\text { (AD) }\end{array}$} & \multicolumn{2}{|c|}{$\begin{array}{c}\text { Sessile Serrated } \\
\text { Adenoma/Polyps } \\
\text { ( } \underline{\text { SSP })}\end{array}$} & \multirow{2}{*}{$\begin{array}{c}\underline{\text { AD }} \text { vs. HP } \\
\text { OR }(95 \% \mathrm{Cl})\end{array}$} & \multirow{2}{*}{$\begin{array}{l}\text { SSP vs. HP } \\
\text { OR }(95 \% \mathrm{Cl})\end{array}$} & \multirow{2}{*}{$\begin{array}{l}\underline{\text { SSP }} \text { vs. } \underline{\mathrm{AD}} \\
\text { OR }(95 \% \mathrm{Cl})\end{array}$} \\
\hline & & $\mathrm{n}$ & OR $(95 \% \mathrm{Cl})$ & $\mathrm{n}$ & OR $(95 \% \mathrm{Cl})$ & $\mathrm{n}$ & OR $(95 \% \mathrm{Cl})$ & & & \\
\hline \multicolumn{11}{|l|}{ Fiber $(\mathbf{g})^{a}$} \\
\hline 2.91-12.88 & 813 & 114 & 1.00 (ref) & 375 & 1.00 (ref) & 57 & 1.00 (ref) & 1.00 (ref) & 1.00 (ref) & 1.00 (ref) \\
\hline $12.88-17.79$ & 811 & 127 & $1.01(0.75,1.38)$ & 319 & $.072(0.58,0.89)$ & 47 & $0.50(0.30,0.82)$ & $0.71(0.51,0.99)$ & $0.49(0.28,0.86)$ & $0.69(0.41,1.15)$ \\
\hline $17.79-24.73$ & 811 & 113 & $0.81(0.57,1.15)$ & 380 & $0.75(0.59,0.94)$ & 37 & $0.64(0.38,1.08)$ & $0.92(0.64,1.34)$ & $0.79(0.43,1.44)$ & $0.85(0.50,1.46)$ \\
\hline$>24.73$ & 811 & 118 & $0.71(0.48,1.05)$ & 428 & $0.65(0.50,0.85)$ & 47 & $0.46(0.19,0.68)$ & $0.92(0.60,1.40)$ & $0.51(0.25,1.04)$ & $0.56(0.29,1.06)$ \\
\hline $\mathbf{P}_{\text {trend }}$ & & & 0.05 & & 0.004 & & 0.006 & 0.96 & 0.17 & 0.12 \\
\hline \multicolumn{11}{|c|}{ Dietary Folate Equivalents $(\mu \mathrm{g})^{\mathrm{a}}$} \\
\hline $63.8-394.7$ & 812 & 119 & 1.00 (ref) & 369 & 1.00 (ref) & 48 & 1.00 (ref) & 1.00 (ref) & 1.00 (ref) & 1.00 (ref) \\
\hline $394.7-572.6$ & 811 & 123 & $0.88(0.64,1.21)$ & 348 & $0.74(0.59,0.91)$ & 48 & $0.99(0.60,1.65)$ & $0.83(0.59,1.18)$ & $1.12(0.64,1.99)$ & $1.35(0.80,2.26)$ \\
\hline $572.6-811.8$ & 811 & 119 & $0.68(0.47,0.97)$ & 380 & $0.64(0.50,0.81)$ & 46 & $0.83(0.47,1.47)$ & $0.94(0.64,1.38)$ & $1.23(0.65,2.32)$ & $1.30(0.73,2.33)$ \\
\hline$>811.8$ & 812 & 111 & $0.53(0.35,0.80)$ & 405 & $0.56(0.43,0.73)$ & 36 & $0.51(0.26,0.98)$ & $1.05(0.68,1.61)$ & $0.96(0.46,2.00)$ & $0.91(0.47,1.78)$ \\
\hline $\mathbf{P}_{\text {trend }}$ & & & $<0.001$ & & $<0.001$ & & 0.03 & 0.62 & 0.94 & 0.69 \\
\hline \multicolumn{11}{|l|}{ Calcium $(\mathbf{m g})^{\mathrm{a}}$} \\
\hline $128.0-595.8$ & 812 & 118 & 1.00 (ref) & 370 & 1.00 (ref) & 53 & 1.00 (ref) & 1.00 (ref) & 1.00 (ref) & 1.00 (ref) \\
\hline $595.8-837.8$ & 811 & 117 & $0.91(0.66,1.26)$ & 342 & $0.80(0.65,1.00)$ & 32 & $0.70(0.42,1.19)$ & $0.88(0.63,1.24)$ & $0.77(0.43,1.38)$ & $0.87(0.51,1.50)$ \\
\hline 837.8-1217 & 811 & 112 & $0.68(0.47,0.99)$ & 390 & $0.68(0.54,0.88)$ & 55 & $0.99(0.56,1.76)$ & $1.00(0.67,1.48)$ & $1.45(0.76,2.76)$ & $1.45(0.81,2.59)$ \\
\hline
\end{tabular}




\begin{tabular}{|c|c|c|c|c|c|c|c|c|c|c|}
\hline \multirow{3}{*}{$\begin{array}{l}\text { Dietary } \\
\text { Intake } \\
\text { (per day) }\end{array}$} & \multicolumn{7}{|c|}{ Case-Control Comparisons } & \multicolumn{3}{|c|}{ Case-Case Comparisons } \\
\hline & \multirow{2}{*}{$\begin{array}{c}\begin{array}{c}\text { No } \\
\text { polyp } \\
\text { Controls }\end{array} \\
\mathrm{n}\end{array}$} & \multicolumn{2}{|r|}{$\begin{array}{l}\text { Hyperplastic } \\
\text { Polyps } \\
\text { (HP) }\end{array}$} & \multicolumn{2}{|c|}{$\begin{array}{l}\frac{\text { Conventional }}{\text { Adenomas }} \\
\text { (AD) }\end{array}$} & \multicolumn{2}{|c|}{$\begin{array}{c}\text { Sessile Serrated } \\
\text { Adenoma/Polyps } \\
\text { ( } \underline{\text { SSP })}\end{array}$} & \multirow{2}{*}{$\begin{array}{c}\underline{\text { AD }} \text { vs. HP } \\
\text { OR }(95 \% \mathrm{Cl})\end{array}$} & \multirow{2}{*}{$\begin{array}{l}\underline{\text { SSP vs. HP }} \\
\text { OR (95\% Cl) }\end{array}$} & \multirow{2}{*}{$\begin{array}{l}\underline{\text { SSP }} \text { vs. } \underline{\text { AD }} \\
\text { OR }(95 \% \mathrm{Cl})\end{array}$} \\
\hline & & $\mathrm{n}$ & OR $(95 \% \mathrm{Cl})$ & $\mathrm{n}$ & OR $(95 \% \mathrm{Cl})$ & $\mathrm{n}$ & OR $(95 \% \mathrm{Cl})$ & & & \\
\hline$>1217$ & 812 & 125 & $0.66(0.44,0.99)$ & 400 & $0.57(0.43,0.75)$ & 38 & $0.54(0.28,1.06)$ & $0.86(0.56,1.33)$ & $0.83(0.40,1.74)$ & $0.96(0.49,1.88)$ \\
\hline $\mathbf{P}_{\text {trend }}$ & & & 0.03 & & $<0.001$ & & 0.13 & 0.64 & 0.90 & 0.88 \\
\hline \multicolumn{11}{|l|}{ Fat $(g)^{a}$} \\
\hline $11.91-48.00$ & 812 & 91 & 1.00 (ref) & 299 & 1.00 (ref) & 38 & 1.00 (ref) & 1.00 (ref) & 1.00 (ref) & 1.00 (ref) \\
\hline $48.00-68.06$ & 810 & 110 & $1.24(0.85,1.80)$ & 293 & $0.85(0.66,1.09)$ & 36 & $1.40(0.77,2.55)$ & $0.69(0.46,1.03)$ & $1.13(0.57,2.22)$ & $1.64(0.88,3.04)$ \\
\hline $68.06-98.16$ & 812 & 126 & $1.23(0.77,1.95)$ & 386 & $0.89(0.66,1.21)$ & 44 & $2.32(1.07,5.04)$ & $1.00(0.44,1.19)$ & $1.89(0.80,4.50)$ & $2.60(1.18,5.76)$ \\
\hline$>98.16$ & 812 & 145 & $1.19(0.69,2.06)$ & 524 & $0.97(0.67,1.39)$ & 60 & $3.09(1.24,7.72)$ & $0.86(0.45,1.46)$ & $2.61(0.94,7.23)$ & $3.20(1.26,8.12)$ \\
\hline $\mathbf{P}_{\text {trend }}$ & & & 0.62 & & 0.99 & & 0.01 & 0.64 & 0.05 & 0.02 \\
\hline \multicolumn{11}{|l|}{ Red Meat $(\mathbf{g})^{a}$} \\
\hline $0-16.06$ & 811 & 73 & 1.00 (ref) & 226 & 1.00 (ref) & 25 & 1.00 (ref) & 1.00 (ref) & 1.00 (ref) & 1.00 (ref) \\
\hline $16.06-38.54$ & 809 & 112 & $1.53(1.10,2.12)$ & 358 & $1.42(1.15,1.76)$ & 33 & $1.42(0.79,2.57)$ & $0.93(0.65,1.33)$ & $0.93(0.48,1.79)$ & $1.00(0.55,1.83)$ \\
\hline $38.54-73.38$ & 807 & 123 & $1.51(1.08,2.10)$ & 360 & $1.36(1.10,1.69)$ & 47 & $2.32(1.32,4.08)$ & $0.90(0.63,1.30)$ & $1.54(0.82,2.90)$ & $1.70(0.95,3.04)$ \\
\hline$>73.38$ & 808 & 163 & $1.68(1.19,2.37)$ & 552 & $1.67(1.34,2.09)$ & 73 & $3.38(1.90,6.00)$ & $1.00(0.69,1.44)$ & $2.02(1.06,3.83)$ & $2.02(1.13,3.63)$ \\
\hline $\mathbf{P}_{\text {trend }}$ & & & 0.009 & & $<0.001$ & & $<0.001$ & 0.93 & 0.006 & 0.003 \\
\hline
\end{tabular}

${ }^{a}$ Derived from multinomial logistic regression models which included all case and controls groups and adjusted for age based on categories (ages 40-49, 50-59, 60-64, and 65+) sex, educational attainment, year of colonoscopy, study site, cigarette use, NSAID use status, and total daily energy intake (divided into quartile categories based on kilocalories/day). 


\section{Evaluation of independent associations}

To evaluate which factors in Tables 2 and 3 were independently associated with polyp risk after mutual adjustment, we conducted an analysis in which factors which were statistically significantly associated with risk of any polyp type were included in a single multinomial logistic $\underline{\text { regression model (Table 4). After adjustment for other factors, SSP risk was no longer }}$ statistically significant for obesity and fiber, folate, and fat intakes although fiber intake was associated with a borderline statistically significant reduced $\underline{\text { SPP. }}$. Conversely, several associations persisted after adjustment. Smoking remained strongly associated with risk of all polyps. NSAID use and red meat intake were associated with SSP risk. 
Table 4: Evaluation of Independent Associations between Modifiable Factors and Polyp Risk, the Tennessee Colorectal Polyp Study

\begin{tabular}{|c|c|c|c|c|c|c|c|}
\hline \multirow[t]{2}{*}{ Factor } & \multirow{2}{*}{$\begin{array}{c}\text { No } \\
\text { polyp } \\
\text { Controls } \\
n\end{array}$} & \multicolumn{2}{|c|}{$\begin{array}{l}\text { Hyperplastic } \\
\text { Polyps } \\
\text { (HP) }\end{array}$} & \multicolumn{2}{|c|}{$\begin{array}{c}\text { Conventional } \\
\text { Adenomas } \\
(\underline{A D})\end{array}$} & \multicolumn{2}{|c|}{$\begin{array}{c}\text { Sessile Serrated } \\
\text { Adenoma/Polyps } \\
\text { (SSP) }\end{array}$} \\
\hline & & $n$ & OR $(95 \% \mathrm{Cl})^{\mathrm{a}}$ & $\mathbf{n}$ & OR $(95 \% \mathrm{Cl})^{\mathrm{a}}$ & $\mathbf{n}$ & OR $(95 \% \mathrm{Cl})^{\mathrm{a}}$ \\
\hline \multicolumn{8}{|c|}{ Cigarette Smoking } \\
\hline Never & 1774 & 147 & 1.00 (ref) & 596 & 1.00 (ref) & 63 & 1.00 (ref) \\
\hline Former & 1126 & 190 & $2.22(1.73,2.85)$ & 550 & $1.24(1.06,1.45)$ & 60 & $1.41(0.94,2.10)$ \\
\hline Current & 343 & 190 & $5.06(3.75,6.82)$ & 355 & $2.68(2.19,3.29)$ & 54 & $4.68(2.99,7.31)$ \\
\hline$P_{\text {trend }}$ & & & $<0.001$ & & $<0.001$ & & $<0.001$ \\
\hline
\end{tabular}

Body Mass Index $\left(\mathrm{kg} / \mathrm{m}^{2}\right)$

$\begin{array}{cccccccc}18.0-24.9 & 1049 & 120 & 1.00(\text { ref }) & 389 & 1.00(\text { ref }) & 49 & 1.00(\text { ref }) \\ 25.0-29.9 & 1224 & 174 & 1.05(0.80,1.37) & 600 & 1.07(0.90,1.27) & 70 & 1.15(0.76,1.74) \\ >30 & 965 & 178 & 1.43(1.09,1.88) & 506 & 1.23(1.02,1.48) & 59 & 1.25(0.80,1.94) \\ \text { Ptrend } & & & & 0.007 & & 0.03 & \end{array}$

\section{Regular NSAID Use}

\begin{tabular}{|c|c|c|c|c|c|c|c|}
\hline Never & 1438 & 197 & 1.00 (ref) & 633 & 1.00 (ref) & 83 & 1.00 (ref) \\
\hline Former & 206 & 29 & $0.96(0.62-1.48)$ & 88 & $0.92(0.69,1.23)$ & 8 & $0.68(0.32,1.46)$ \\
\hline Current & 1593 & 228 & $0.90(0.73,1.13)$ & 679 & $0.77(0.66,0.89)$ & 75 & $0.68(0.48,0.96)$ \\
\hline Prend & & & 0.36 & & $<0.001$ & & 0.03 \\
\hline
\end{tabular}




\begin{tabular}{|c|c|c|c|c|c|c|c|}
\hline \multirow[t]{2}{*}{ Factor } & \multirow{2}{*}{$\begin{array}{c}\text { No } \\
\text { polyp } \\
\text { Controls } \\
n\end{array}$} & \multicolumn{2}{|c|}{$\begin{array}{l}\text { Hyperplastic } \\
\text { Polyps } \\
\text { (HP) }\end{array}$} & \multicolumn{2}{|c|}{$\begin{array}{c}\text { Conventional } \\
\text { Adenomas } \\
(\underline{A D})\end{array}$} & \multicolumn{2}{|c|}{$\begin{array}{c}\text { Sessile Serrated } \\
\text { Adenoma/Polyps } \\
\text { (SSP) }\end{array}$} \\
\hline & & $\mathbf{n}$ & OR $(95 \% \mathrm{Cl})^{\mathrm{a}}$ & $\mathbf{n}$ & OR $(95 \% \mathrm{Cl})^{\mathrm{a}}$ & $\mathbf{n}$ & OR $(95 \% \mathrm{Cl})^{a}$ \\
\hline \multicolumn{8}{|c|}{ Fiber Intake (g/day) } \\
\hline $2.91-12.88$ & 813 & 114 & 1.00 (ref) & 375 & 1.00 (ref) & 57 & 1.00 (ref) \\
\hline $12.88-17.79$ & 811 & 127 & $1.16(0.83,1.63)$ & 319 & $0.83(0.66,1.05)$ & 37 & $0.47(0.27,0.82)$ \\
\hline $17.79-24.73$ & 811 & 113 & $1.05(0.70,1.58)$ & 380 & $0.95(0.73,1.24)$ & 47 & $0.63(0.34,1.17)$ \\
\hline $24.73-126.3$ & 811 & 118 & $1.09(0.68,1.76)$ & 428 & $0.93(0.68,1.28)$ & 37 & $0.47(0.22,1.01)$ \\
\hline$P_{\text {trend }}$ & & & 0.85 & & 0.92 & & 0.11 \\
\hline \multicolumn{8}{|c|}{ Dietary Folate Equivalents Intake ( $\mu$ g/day) } \\
\hline $63.81-394.7$ & 812 & 119 & 1.00 (ref) & 369 & 1.00 (ref) & 48 & 1.00 (ref) \\
\hline $394.7-572.6$ & 811 & 123 & $0.86(0.60,1.23)$ & 348 & $0.82(0.64,1.04)$ & 48 & $1.37(0.78,2.43)$ \\
\hline $572.6-811.8$ & 811 & 119 & $0.70(0.45,1.07)$ & 380 & $0.74(0.55,0.98)$ & 46 & $1.26(0.63,2.53)$ \\
\hline $811.8-4542$ & 812 & 111 & $0.57(0.34,0.95)$ & 405 & $0.71(0.50,0.99)$ & 36 & $1.00(0.44,2.29)$ \\
\hline$P_{\text {trend }}$ & & & 0.03 & & 0.05 & & 0.9 \\
\hline \multicolumn{8}{|c|}{ Dietary Calcium Intake (mg/day) } \\
\hline $128.0-595.8$ & 812 & 118 & 1.00 (ref) & 370 & 1.00 (ref) & 53 & 1.00 (ref) \\
\hline $595.8-837.8$ & 811 & 117 & $0.98(0.70,1.37)$ & 342 & $0.91(0.72,1.14)$ & 32 & $0.74(0.42,1.29)$ \\
\hline $837.8-1217$ & 811 & 112 & $0.83(0.56,1.24)$ & 390 & $0.81(0.61,1.06)$ & 55 & $1.10(0.59,2.05)$ \\
\hline $1217-6880$ & 812 & 125 & $0.86(0.55,1.35)$ & 400 & $0.68(0.50,0.92)$ & 38 & $0.70(0.33,1.45)$ \\
\hline$P_{\text {trend }}$ & & & 0.44 & & 0.01 & & 0.47 \\
\hline
\end{tabular}

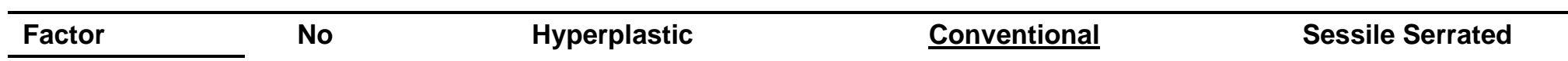




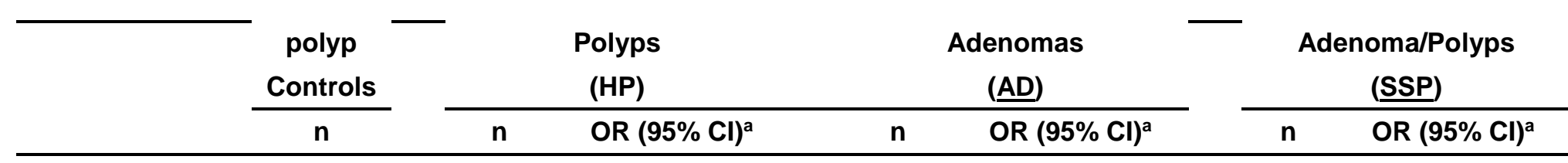

\section{Total Fat Intake (g/day)}

$\begin{array}{llcccccc}11.91-48.00 & 812 & 91 & 1.00(\text { ref }) & 299 & 1.00(\text { ref }) & 38 & 1.00(\text { ref }) \\ 48.00-68.06 & 810 & 110 & 1.12(0.76,1.64) & 293 & 0.80(0.62,1.04) & 36 & 1.30(0.70,2.42) \\ 68.06-98.16 & 812 & 126 & 0.98(0.60,1.59) & 386 & 0.76(0.55,1.05) & 44 & 1.79(0.79,4.04) \\ 98.16-377.4 & 812 & 145 & 0.89(0.49,1.60) & 524 & 0.80(0.55,1.19) & 60 & 2.15(0.81,5.69) \\ P_{\text {trend }} & & & & 0.58 & & 0.32 & \end{array}$

\section{Red Meat Intake (g/day)}

\begin{tabular}{|c|c|c|c|c|c|c|c|}
\hline $0.0-16.07$ & 811 & 73 & 1.00 (ref) & 226 & 1.00 (ref) & 25 & 1.00 (ref) \\
\hline $16.07-38.54$ & 809 & 112 & $1.46(1.04,2.03)$ & 358 & $1.38(1.11,1.71)$ & 33 & $1.30(0.71,2.36)$ \\
\hline $38.54-73.88$ & 807 & 123 & $1.38(0.98,1.96)$ & 360 & $1.28(1.03,1.61)$ & 47 & $1.90(1.06,3.41)$ \\
\hline $73.88-625.8$ & 808 & 163 & $1.48(1.03,2.14)$ & 552 & $1.53(1.21,1.94)$ & 73 & $2.59(1.41,4.74)$ \\
\hline trend & & & 0.08 & & 0.002 & & $<0.001$ \\
\hline
\end{tabular}

${ }^{a}$ Derived from multinomial logistic regression models which included all case and controls groups and adjusted for age (40-49, 50-59, 60-64, and 65+ years of age), sex, educational attainment, year of colonoscopy, study site, and total daily energy intake (divided into quartile categories based on kilocalories/day). Additionally adjusted for all variables within the table. 


\section{DISCUSSION}

This analysis assesses modifiable lifestyle risk factors in a screening colonoscopy-age population to evaluate risk factors for SSP and to compare them with other common colorectal polyps. Given the recent identification within the past 1-2 decades of SSPs as a CRC precursor, we are still in the infancy of understanding the etiology of these lesions and which risk factors may be associated with these polyps. With their importance in the pathways' underlying progression to cancer and the relative difficulty in identification on colonoscopy, finding ways to assess risk in a population are of utmost importance. This is the first study to evaluate dietary intake with risk for SSPs and one of the largest epidemiologic studies to date of SSPs. We newly found that red meat, fat, and fiber intakes were associated with $\underline{\text { SSP }}$ risk, and we also confirmed previous findings of associations with cigarette smoking and with NSAID use, and a lack of association with alcohol use.

Unlike a consistent association with polyp risk [14,17], cigarette smoking has been modestly and inconsistently associated with CRC risk [18]. One possible reason for the inconsistency in past studies is a mixing of the types of CRC tumors which have different associations. Recent studies have more consistently identified smoking as a risk factor of MSI-high or CIMP-high CRC tumors which are part of the serrated pathway $[19,20]$. Indeed, cigarette smoking is strongly and consistently associated with risk of serrated polyps, including in this study [3,1014,21]. Smoking cessation has many benefits for health and we found cessation as short as 10 years was associated with decreased risk of all polyps compared to current smokers. Further, after cessation for more than 20 years, risk was similar to never smokers. This relationship was particularly strong for SSPs vs. $\underline{\text { ADs. }}$.

NSAIDs and aspirin use may be an approach for colorectal neoplasia prevention; however, very little is known regarding NSAIDs and their association with SSP risk [10,13,22,23]. A previous 
study of serrated polyps found an inverse association between aspirin use and serrated polyp risk which was particularly strong for proximal lesions [10]. In the only previous study to evaluate SSP risk, regular NSAID use was associated with reduced risk. We also observed this [13]. We also found the reduction in risk associated with more than 10 years of use was stronger for $\underline{\text { SSPS }}$ than for $\underline{\mathrm{ADS}}$. The absence of an association with HP risk and the presence of an association with $\underline{\text { SPP }}$ risk may provide insight into the etiology of SSPs and may be a distinguishing factor in inhibiting transition from HP to SSP. Thus, NSAID use may hold promise as a chemopreventive strategy for SSPs and should be evaluated in future studies.

Body composition and exercise are well studied modifiable factors evaluated in $\underline{A D}$ and CRC risk $[24,25]$. An association between SSP risk and obesity is currently equivocal [10-13]. Although we observed a statistically significant association between BMI-defined obesity and colorectal polyp risk in all case groups, after adjustment for other factors, a statistically significant association was no longer observed for SSP risk. Interestingly, no association was observed for physical activity measures, including a measure of intensity and duration (MET hours). Both of these findings are consistent with a previous study which found no association between either BMI or hours of exercise with SSPs risk [13].

Dietary fiber has been speculated to protect against polyp formation by bulking the stool and increasing transit time, which may decrease the surface area of the colon exposed to carcinogenic toxins and bile acids within fecal matter [31]. Although we initially observed decreased risks of adenomas with fiber intake, these associations did not persist in subsequent models after adjustment for other risk factors. However, a suggestive borderline significant inverse association was observed with highest fiber intake and SSP risk. Future studies with a larger sample size are needed to confirm this finding. Likewise, both calcium and folate intakes initially appeared to be associated with decreased risk of SSPs However, the associations disappeared after adjustment for other factors. Thus, this study does not support a strong 
relationship between calcium or folate intakes with SSP risk (although these factors should be evaluated in future larger studies). This result is also consistent with the findings from recent randomized trials in which supplementation of calcium or folic acid have not successfully decreased risk of $\underline{A D}$ recurrence $[32-35]$.

Red meat intake is consistently reported as a risk factor for CRC and colorectal adenomas $[36,37]$, although it has not previously been known whether an association exists between red meat intake and SSP risk. Risk of MSI-high CRC, for which $\underline{\text { SPPs }}$ are the presumed precursor lesion, is increased with well-done red meat intake, suggesting a possible role of red meat intake in SSP risk [38]. Consistent with this finding, we found, for the first time, that high consumption of red meat was strongly associated with SSP risk. Interestingly, we also found that higher dietary fat intake was associated with risk of SSPs but not ADs or HPs; however, this relationship did not maintain statistical significance after adjustment for other risk factors. These included red meat intake, which may have been due to our sample size, over adjustment, or may suggest that the fat intake association is a potential measure of red meat intake. Previous studies of serrated polyps have observed an association between high fat diet and serrated polyp risk, although this was not specific to SSPs [10]. The potential mechanism behind an association is unclear, and further studies are needed.

There are several strengths within this study. To date, it is one of only two large studies

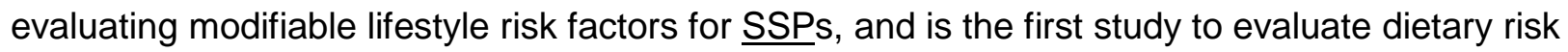
factors for SSPs [13]. We were able to rigorously standardize the diagnosis of all polyps regardless of initial clinical diagnosis using recently developed standards for HPS, $\underline{A D s}$, and $\underline{\text { SSPs }}$ and our observed prevalence of SSPs was consistent with recent prevalence studies $[3,39]$. We were able to comprehensively evaluate several different modifiable factors. 
There are also weaknesses in this study, which we attempted to limit. As with all case-control studies, we cannot exclude the possibility of recall bias although it may have been minimized because colorectal polyps are a benign diagnosis and the data collection period was short. $\underline{\text { Recent studies have indicated that several factors may contribute to detection rates of polyps }}$ including quality of the bowel cleansing and withdrawal time[40]. We did not collect data on these factors and so cannot exclude the possibility of missed polyps which may have resulted in

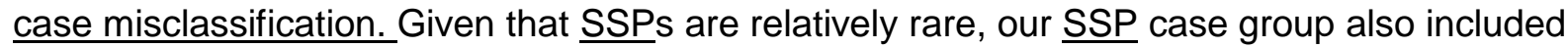
individuals with synchronous $\underline{A D s}(43 \%)$, which could potentially have affected the results if the risk factor was associated with $\underline{\mathrm{ADs}}$ and not SSAs. However, we also observed associations that were only present for SSP risk, suggesting that we were able to evaluate risk factors for $\underline{\text { SSP. }}$. We did perform sensitivity analysis by examining individuals $\underline{\text { SPP }}$ who did not have any Ads (supplemental tables). Although this diminished statistical power, we observed very similar results for all factors analyzed as we observed when including individuals with $\underline{\text { ADs }}$ in the SSP case group, thus, indicating that the presence of an $\underline{A D}$ was not likely driving the observed associations. We may have failed to detect an association because statistical power in some of $\underline{\text { the subgroup analyses could have been limited. Thus, future larger studies are needed. }}$ Although this study included individuals with a wide range of characteristics and behaviors and we observed associations which both increased or decreased risk, we cannot exclude the possibility that individuals who receive colonoscopies are different in ways from individuals who do not receive colonoscopies which may affect the observed associations in an unknown $\underline{\text { manner. }}$

In summary, this study provides an extensive evaluation of lifestyle risk factors for SSPs and a

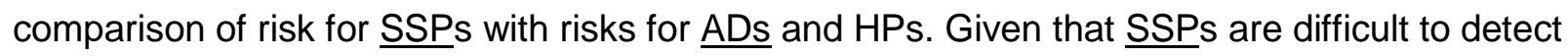
and fully remove on endoscopic screening and may accelerate to a dysplastic state quicker than $\underline{\mathrm{ADs}}[4,8,9,41-43]$, primary prevention of SSPs through lifestyle modification may be an 
important strategy. The study found that many of the same risk factors are shared between $\underline{A D s}, \mathrm{HPs}$, and SSPs. Thus, preventive efforts to reduce risk factors in $\underline{\mathrm{ADs}}$ may also be applicable to SSPs. The study also found some differences in risk factors between the polyp types. Larger studies of SSPs will be needed to confirm these findings and future studies should also evaluate potential interactions of these risk factors with genetic or molecular risk factors, as well as preventive strategies that may be unique to SSPs.

\section{ACKNOWLEDGEMENTS}

The authors wish to thank Dr. Kay Washington for her assistance with sessile serrated polyp diagnosis. The authors also wish to thank the many research staff and investigators who have contributed to the Tennessee Colorectal Polyp Study. Finally, the authors thank the study participants who contributed their time and biospecimens for research.

\section{CONTRIBUTORS}

RMN, WZ, and MJS contributed to study conception, design, and supervision. TS, WES, RMN, and MJS contributed to acquisition of data. TS and MJS provided administrative, technical, or material support. JRD, ZZ, HGC, WZ, and MJS contributed to analysis and interpretation of data. All authors contributed to writing, review, and/or revision of the manuscript and approved the final manuscript. MJS is the guarantor of the submitted manuscript.

\section{FUNDING}

This study was supported by grants P50CA950103, R01CA97386, and K07CA122451. JRD was supported by the Molecular and Genetic Epidemiology of Cancer fellowship (R25CA160056). Surveys and sample collection and processing for this study were conducted by the Survey and Biospecimen Shared Resource, which is supported in part by P30CA68485. The content of this paper is solely the responsibility of the authors and does not necessarily 
represent the official views of the National Cancer Institute or the National Institutes of Health. A portion of the participants were studied as the result of resources and the use of facilities at the VA Tennessee Valley Healthcare System.

\section{COMPETING INTERESTS}

No authors of this manuscript have any conflicts of interest to report.

\section{ETHICS APPROVAL}

Written informed consent was obtained from all study participants, and the study protocol was approved by the Institutional Review Board at each study site 


\section{REFERENCES}

1 Fearon ER, Vogelstein B. A genetic model for colorectal tumorigenesis. Cell 1990;61:759_ 67.

2 Snover DC. Update on the serrated pathway to colorectal carcinoma. Hum Pathol 2011;42:1-10. doi:10.1016/j.humpath.2010.06.002

3 Rex DK, Ahnen DJ, Baron JA, et al. Serrated lesions of the colorectum: review and recommendations from an expert panel. Am J Gastroenterol 2012;107:1315-1329, 1330. doi:10.1038/ajg.2012.161

4 Crockett SD, Snover DC, Ahnen DJ, et al. Sessile serrated adenomas: an evidence-based guide to management. Clin Gastroenterol Hepatol 2015;13:11-26.e1.

doi:10.1016/j.cgh.2013.10.035

5 IJspeert JEG, Bevan R, Senore C, et al. Detection rate of serrated polyps and serrated polyposis syndrome in colorectal cancer screening cohorts: a European overview. Gut Published Online First: 24 February 2016. doi:10.1136/gutjnl-2015-310784

6 Hetzel JT, Huang CS, Coukos JA, et al. Variation in the detection of serrated polyps in an average risk colorectal cancer screening cohort. Am J Gastroenterol 2010;105:2656-64. doi:10.1038/ajg.2010.315

7 Bailey CE, Hu C-Y, You YN, et al. Increasing disparities in the age-related incidences of colon and rectal cancers in the United States, 1975-2010. JAMA Surg 2015;150:17-22. doi:10.1001/jamasurg.2014.1756

8 Samadder NJ, Curtin K, Tuohy TMF, et al. Characteristics of missed or interval colorectal cancer and patient survival: a population-based study. Gastroenterology 2014;146:950-60. doi:10.1053/j.gastro.2014.01.013

9 Singh S, Singh PP, Murad MH, et al. Prevalence, risk factors, and outcomes of interval colorectal cancers: a systematic review and meta-analysis. Am J Gastroenterol 2014;109:1375-89. doi:10.1038/ajg.2014.171

10 Wallace K, Grau MV, Ahnen D, et al. The association of lifestyle and dietary factors with the risk for serrated polyps of the colorectum. Cancer Epidemiol Biomarkers Prev 2009;18:2310-7. doi:10.1158/1055-9965.EPI-09-0211

11 Anderson JC, Rangasamy P, Rustagi T, et al. Risk factors for sessile serrated adenomas. J Clin Gastroenterol 2011;45:694-9. doi:10.1097/MCG.0b013e318207f3cf

12 Rustagi T, Rangasamy P, Myers M, et al. Sessile serrated adenomas in the proximal colon are likely to be flat, large and occur in smokers. World J Gastroenterol 2013;19:5271-7. doi:10.3748/wjg.v19.i32.5271

13 Burnett-Hartman AN, Passarelli MN, Adams SV, et al. Differences in epidemiologic risk factors for colorectal adenomas and serrated polyps by lesion severity and anatomical site. Am J Epidemiol 2013;177:625-37. doi:10.1093/aje/kws282 
14 Figueiredo JC, Crockett SD, Snover DC, et al. Smoking-associated risks of conventional adenomas and serrated polyps in the colorectum. Cancer Causes Control 2015;26:377-86. doi:10.1007/s10552-014-0513-0

15 Shin A, Shrubsole MJ, Ness RM, et al. Meat and meat-mutagen intake, doneness preference and the risk of colorectal polyps: the Tennessee Colorectal Polyp Study. Int J Cancer 2007;121:136-42. doi:10.1002/ijc.22664

16 Signorello LB, Munro HM, Buchowski MS, et al. Estimating nutrient intake from a food frequency questionnaire: incorporating the elements of race and geographic region. $\mathrm{Am} \mathrm{J}$ Epidemiol 2009;170:104-11. doi:10.1093/aje/kwp098

17 Botteri E, lodice S, Raimondi S, et al. Cigarette smoking and adenomatous polyps: a metaanalysis. Gastroenterology 2008;134:388-95. doi:10.1053/j.gastro.2007.11.007

18 Liang PS, Chen T-Y, Giovannucci E. Cigarette smoking and colorectal cancer incidence and mortality: systematic review and meta-analysis. Int J Cancer 2009;124:2406-15. doi:10.1002/ijc.24191

19 Weisenberger DJ, Levine AJ, Long TI, et al. Association of the colorectal CpG island methylator phenotype with molecular features, risk factors, and family history. Cancer Epidemiol Biomarkers Prev 2015;24:512-9. doi:10.1158/1055-9965.EPI-14-1161

20 Limsui D, Vierkant RA, Tillmans LS, et al. Cigarette smoking and colorectal cancer risk by molecularly defined subtypes. J Natl Cancer Inst 2010;102:1012-22.

doi:10.1093/jnci/djq201

21 Shrubsole MJ, Wu H, Ness RM, et al. Alcohol drinking, cigarette smoking, and risk of colorectal adenomatous and hyperplastic polyps. Am J Epidemiol 2008;167:1050-8. doi:10.1093/aje/kwm400

22 Cole BF, Logan RF, Halabi S, et al. Aspirin for the chemoprevention of colorectal adenomas: meta-analysis of the randomized trials. J Natl Cancer Inst 2009;101:256-66. doi:10.1093/jnci/djn485

23 Murff HJ, Shrubsole MJ, Chen Z, et al. Nonsteroidal anti-inflammatory drug use and risk of adenomatous and hyperplastic polyps. Cancer Prev Res (Phila) 2011;4:1799-807. doi:10.1158/1940-6207.CAPR-11-0107

24 Karahalios A, English DR, Simpson JA. Weight change and risk of colorectal cancer: a systematic review and meta-analysis. Am J Epidemiol 2015;181:832-45. doi:10.1093/aje/kwu357

25 Ben Q, An W, Jiang Y, et al. Body mass index increases risk for colorectal adenomas based on meta-analysis. Gastroenterology 2012;142:762-72. doi:10.1053/j.gastro.2011.12.050

26 Giovannucci E, Ascherio A, Rimm EB, et al. Physical activity, obesity, and risk for colon cancer and adenoma in men. Ann Intern Med 1995;122:327-34. 
27 Calle EE, Rodriguez C, Walker-Thurmond K, et al. Overweight, obesity, and mortality from cancer in a prospectively studied cohort of U.S. adults. N Engl J Med 2003;348:1625-38. doi:10.1056/NEJMoa021423

28 Murphy TK, Calle EE, Rodriguez C, et al. Body mass index and colon cancer mortality in a large prospective study. Am J Epidemiol 2000;152:847-54.

29 Renehan AG, Tyson M, Egger M, et al. Body-mass index and incidence of cancer: a systematic review and meta-analysis of prospective observational studies. Lancet 2008;371:569-78. doi:10.1016/S0140-6736(08)60269-X

30 Okabayashi $\mathrm{K}$, Ashrafian $\mathrm{H}$, Hasegawa $\mathrm{H}$, et al. Body mass index category as a risk factor for colorectal adenomas: a systematic review and meta-analysis. Am J Gastroenterol 2012;107:1175-1185; quiz 1186. doi:10.1038/ajg.2012.180

31 Alberts DS, Ritenbaugh C, Story JA, et al. Randomized, double-blinded, placebo-controlled study of effect of wheat bran fiber and calcium on fecal bile acids in patients with resected adenomatous colon polyps. J Natl Cancer Inst 1996;88:81-92.

32 Cole BF, Baron JA, Sandler RS, et al. Folic acid for the prevention of colorectal adenomas: a randomized clinical trial. JAMA 2007;297:2351-9. doi:10.1001/jama.297.21.2351

33 Logan RFA, Grainge MJ, Shepherd VC, et al. Aspirin and folic acid for the prevention of recurrent colorectal adenomas. Gastroenterology 2008;134:29-38.

doi:10.1053/j.gastro.2007.10.014

$34 \mathrm{Wu}$ K, Platz EA, Willett WC, et al. A randomized trial on folic acid supplementation and risk of recurrent colorectal adenoma. Am J Clin Nutr 2009;90:1623-31.

doi:10.3945/ajen.2009.28319

35 Baron JA, Barry EL, Mott LA, et al. A Trial of Calcium and Vitamin D for the Prevention of Colorectal Adenomas. N Engl J Med 2015;373:1519-30. doi:10.1056/NEJMoa1500409

36 Lippi G, Mattiuzzi C, Cervellin G. Meat consumption and cancer risk: a critical review of published meta-analyses. Crit Rev Oncol Hematol 2016;97:1-14.

doi:10.1016/j.critrevonc.2015.11.008

37 Carr PR, Walter V, Brenner H, et al. Meat subtypes and their association with colorectal cancer: Systematic review and meta-analysis. Int J Cancer 2016;138:293-302.

doi:10.1002/ijc.29423

$38 \mathrm{Wu}$ AH, Shibata D, Yu MC, et al. Dietary heterocyclic amines and microsatellite instability in colon adenocarcinomas. Carcinogenesis 2001;22:1681-4.

39 Abdeljawad K, Vemulapalli KC, Kahi CJ, et al. Sessile serrated polyp prevalence determined by a colonoscopist with a high lesion detection rate and an experienced pathologist. Gastrointest Endosc 2015;81:517-24. doi:10.1016/j.gie.2014.04.064

40 Schoenfeld PS, Cohen J. Quality indicators for colorectal cancer screening for colonoscopy(.). Tech Gastrointest Endosc 2013;15:59-68. doi:10.1016/j.tgie.2013.02.005 
41 Lash RH, Genta RM, Schuler CM. Sessile serrated adenomas: prevalence of dysplasia and carcinoma in 2139 patients. J Clin Pathol 2010;63:681-6. doi:10.1136/jcp.2010.075507

42 Sawhney MS, Farrar WD, Gudiseva S, et al. Microsatellite instability in interval colon cancers. Gastroenterology 2006;131:1700-5. doi:10.1053/j.gastro.2006.10.022

43 Burnett-Hartman AN, Newcomb PA, Phipps Al, et al. Colorectal endoscopy, advanced adenomas, and sessile serrated polyps: implications for proximal colon cancer. Am J Gastroenterol 2012;107:1213-9. doi:10.1038/ajg.2012.167 